Publicacions Matemàtiques, Vol 37 (1993), 317-334.

\title{
SHAPE THEORY INTRINSICALLY
}

\author{
ZVONKO ČERIN
}

\begin{abstract}
We prove in this paper that the category $\mathcal{H} M$ whose objects are topological spaces and whose morphisms are homotopy classes of multi-nets is naturally equivalent to the shape category $S h$. The description of the category $\mathcal{H} M$ was given earlier in the article "Shape via multi-nets". We have shown there that $\mathcal{H} M$ is naturally equivalent to $S h$ only on a rather restricted class of spaces. This class includes all compact metric spaces where a similar intrinsic description of the shape category using multi-valued functions was given by José M. R. Sanjurjo in [5] and [6].
\end{abstract}

\section{Preliminaries for the description of the category $\mathcal{H M}$}

In this section we shall collect definitions and results from [2] that are required for the description of the category $\mathcal{H M}$.

\section{Normal covers.}

Let $\hat{Y}$ denote the collection of all normal covers of a topological space $Y$ [1]. With respect to the refinement relation $>$ the set $\hat{Y}$ is a directed set. Two normal covers $\sigma$ and $\tau$ of $Y$ are equivalent provided $\sigma>\tau$ and $\tau>\sigma$. In order to simplify our notation we denote a normal cover and it's equivalence class by the same symbol. Consequently, $\hat{Y}$ also stands for the associated quotient set.

Let $\hat{Y}$ denote the collection of all finite subsets $c$ of $\hat{Y}$ which have a unique (with respect to the refinement relation) maximal element $\bar{c} \in \hat{Y}$. We consider $\tilde{Y}$ ordered by the inclusion relation and regard $\hat{Y}$ as a subset of single-element subsets of $\hat{Y}$. Notice that $\tilde{Y}$ is a cofinite directed set.

Let $\sigma \in \hat{Y}$. Let $\sigma^{*}$ denote the set of all normal covers $\tau$ of $Y$ such that the star $s t(\tau)$ of $\tau$ refines $\sigma$. Similarly, for a natural number $n, \sigma^{* n}$ denotes the set of all normal covers $\tau$ of $Y$ such that the $n$-th star $s t^{n}(\tau)$ of $\tau$ refines $\sigma$. 


\section{Multi-valued functions.}

Let $X$ and $Y$ be topological spaces. By a multi-valued function $F$ : $X \rightarrow Y$ we mean a rule which associates a non-empty subset $F(x)$ of $Y$ to every point $x$ of $X$. Let $M(X, Y)$ denote all multi-valued functions from $X$ into $Y$.

Let $F: X \rightarrow Y$ be a multi-valued function and let $\alpha \in \hat{X}$ and $\gamma \in \hat{Y}$. We shall say that $F$ is an $(\alpha, \gamma)$-function provided for cvery $A \in \alpha$ there is a $C_{A} \in \gamma$ with $F(A) \subset C_{A}$. On the other hand, $F$ is $\gamma$-small provided there is an $\alpha \in \hat{X}$ such that $F$ is an $(\alpha, \gamma)$-function.

Let $F, G: X \rightarrow Y$ be multi-valued functions and let $\gamma \in \hat{Y}$. We shall say that $F$ and $G$ are $\gamma$-close and we write $F \stackrel{\gamma}{=} G$ provided for every $x \in X$ there is a $C_{x} \in \gamma$ with $F(x) \cup G(x) \subset C_{x}$. How one defines the notion " $(\alpha, \gamma)$-close" is now obvious.

Let $F, G: X \rightarrow Y$ be multi-valued functions between topological spaces and let $\gamma$ be a normal cover of the space $Y$. We shall say that $F$ and $G$ are $\gamma$-homotopic and write $F \stackrel{\gamma}{\simeq} G$ provided there is a $\gamma$-small multi-valued function $H$ from the product $X \times I$ of $X$ and the unit segment $I=[0,1]$ into $Y$ such that $F(x) \subset H(x, 0)$ and $G(x) \subset H(x, 1)$ for every $x \in X$. We shall say that $H$ is a $\gamma$-homotopy that joins $F$ and $G$ or that it realizes the relation (or homotopy) $F \stackrel{\chi}{\simeq} G$.

Lemma 1. Let $F, G, H: X \rightarrow Y$ be multi-valued functions. Let $\sigma \in \hat{Y}$ and $\tau \in \sigma^{*}$. If $F \stackrel{\tau}{\simeq} G$ and $G \stackrel{\tau}{\simeq} H$, then $F \stackrel{\sigma}{\simeq} H$.

\section{Multi-nets.}

Let $X$ and $Y$ be topological spaces. By a multi-net from $X$ into $Y$ we shall mcan a collection $\varphi=\left\{F_{c} \mid c \in \hat{Y}\right\}$ of multi-valucd functions $F_{c}: X \rightarrow Y$ such that for every $\gamma \in \hat{Y}$ there is a $c \in \tilde{Y}$ with $F_{d} \stackrel{\gamma}{\simeq} F_{c}$ for every $d>c$. We use functional notation $\varphi: X \rightarrow Y$ to indicate that $\varphi$ is a multi-net from $X$ into $Y$. Let $M N(X, Y)$ denote all multi-nets $\varphi: X \rightarrow Y$.

Two multi-nets $\varphi=\left\{F_{c}\right\}$ and $\psi=\left\{G_{c}\right\}$ between topological spaces $X$ and $Y$ are homotopic provided for every $\gamma \in \hat{Y}$ there is a $c \in \tilde{Y}$ such that $F_{d} \stackrel{\gamma}{=} G_{d}$ for every $d>c$.

It follows from Lemma 1 that the relation of homotopy is an equivalence relation on the set $M N(X, Y)$. The homotopy class of a multi-net $\varphi$ is denoted by $[\varphi]$ and the set of all homotopy classes by $\mathcal{H} M(X, Y)$. 


\section{Description of the category $\mathcal{H M}$}

\section{Composition of homotopy classes.}

Our goal now is to define a composition for homotopy classes of multinets.

Let $\varphi=\left\{F_{c}\right\}: X \rightarrow Y$ be a multi-net. For every $c \in \bar{Y}$ there is an $\bar{f}(c) \in \tilde{Y}$ such that for all $d, e>\bar{f}(c)$ there is a normal cover $\bar{f}(c, d, e)$ of $X \times I$ and an $(\bar{f}(c, d, e), \bar{c})$-map joining $F_{d}$ and $F_{e}$.

Let $\mathcal{C}=\{(c, d, e) \mid c \in \tilde{Y}, d, e>\bar{f}(c)\}$. Then $C$ is a subset of $\tilde{Y} \times \tilde{Y} \times \dot{Y}$ that becomes a cofinite directed set when we define that $(c, d, e)>\left(c^{\prime}, d^{\prime}, e^{\prime}\right)$ iff $c>c^{\prime}, d>d^{\prime}$, and $e>e^{\prime}$.

Now, let $f: \tilde{Y} \rightarrow \tilde{Y}$ be an increasing function such that $f(c)>\bar{f}(c), c$ for every $c \in \tilde{Y}$. We shall use the same notation $f$ for an increasing function $f: \mathcal{C} \rightarrow X \hat{\times} I$ such that $f(c, d, e)>\bar{f}(c, d, e)$ for every $(c, d, e) \in \mathcal{C}$. Let $(c, d, e) \in \mathcal{C}$. For the normal cover $f(c, d, e)$ of $X \times I$, by $[3$, p. 358], there is a normal cover $\varepsilon=\hat{f}(c, d, e)$ of $X$ and a function $r=f(c, d, e): \varepsilon \rightarrow\{2,3,4, \ldots\}$ such that every set $E \times[(i-1) / r E,(i+1) / r E]$, where $E \in \varepsilon$ and $i=1,2, \ldots, r E-1$, is contained in a momber of $f(c, d, e)$.

Let $\tilde{f}: \mathcal{C} \rightarrow \hat{X}$ be an increasing function with $\tilde{f}(c, d, e)>\hat{f}(c, d, e)$ for every $(c, d, e) \in \mathcal{C}$. We shall usc the shorter notation $\tilde{f}(c)$ and $f(c)$ for the covers $f(c, f(c), f(c))$ and $f(c, f(c), f(c))$. In [2, Claim 1], the following lemma was proved.

Lemma 2. There is an increasing function $f^{*}: \vec{Y} \rightarrow \hat{X}$ such that

(1) $f^{*}(c)>\vec{f}(c)$ for every $c \in \bar{Y}$, and

(2) $f^{*}$ is cofinal in $\bar{f}, i$. e, for every $(c, d, e) \in \mathcal{C}$ there is an $m \in \dot{Y}$ with $f^{*}(m)>\tilde{f}(c, d, e)$.

The above discussion shows that every multi-nct $\varphi: X \rightarrow Y$ determines eight functions denoted by $\bar{f}, f, \hat{f}, \bar{f}$, and $f^{*}$. With the help of these functions we shall define the composition of homotopy classes of multi-nets as follows.

Let $\varphi=\left\{F_{c}\right\}: X \rightarrow Y$ and $\psi=\left\{G_{u}\right\}: Y \rightarrow Z$ be multi-nets. Let. $\chi=\left\{H_{u}\right\}$, where $H_{u}=G_{g(u)} \circ F_{f\left(\left\{g^{*}(u)\right\}\right)}$ for cvery $u \in \tilde{Z}$.

It was proved in [2] that the collection $\chi$ is a multi-net from $X$ into $Z$.

We now define the composition of homotopy classes of multi-nets by the rule $\left[\left\{G_{u}\right\}\right] \circ\left[\left\{F_{c}\right\}\right]=\left[\left\{G_{g(u)} \circ F_{f\left(\left\{g^{*}(u)\right\}\right)}\right\}\right]$. The composition of homotopy classes of multi-nets is well-defined and associative (see [2]). 


\section{The category $\mathcal{H} M$.}

For a topological space $X$, let $\iota^{X}=\left\{I_{a}\right\}: X \rightarrow X$ be the identity multi-net defined by $I_{a}=i d_{X}$ for every $a \in \tilde{X}$. It is easy to show that for every multi-net $\varphi: X \rightarrow Y$ the following relations hold:

$$
[\varphi] \circ\left[\iota^{X}\right]=[\varphi]=\left[\iota^{Y}\right] \circ[\varphi] .
$$

It was shown in $[2]$ that the topological spaces as objects, the homotopy classes of multi-nets as morphisms, the homatopy classes $\left[\iota^{x}\right]$ as identities, and the above composition of homotopy classes form the category $\mathcal{H} M$.

There is an obvious functor $J$ from the category Top of topological spaces and continuous maps into the category $\mathcal{H M}$. On objects the functor $J$ is the identity while on morphisms it associates to a map $f: X \rightarrow Y$ the homotopy class of a multi-net $\underline{f}=\left\{F_{c}\right\}: X \rightarrow Y$, where $F_{c}=f$ for every $c \in \tilde{Y}$.

\section{Statement of the main theorem}

Our main result can be stated as follows. Let $S h$ be the shape category of arbitrary topological spaces and let $S: T o p \rightarrow \mathcal{S} h$ be the shape functor [4].

Theorem. There is a functor $\theta$ from the category $\mathcal{H} M$ into the shape category $S h$ which is an isomorphism of categories and such that $S=$ $\theta \circ J$.

\section{Preliminaries for the description of the functor $\theta$}

\section{Cofinite Čech system.}

With every space $X$ one can associate an inverse system $\mathcal{X}=$ $\left\{X_{c},\left[p_{d}^{c}\right], \tilde{X}\right\}$, called the cofinite Cech system of $X$, where $X_{c}=|N(\vec{c})|$ is the nerve of $\bar{c}$ and $\left[p_{d}^{c}\right]$, for $d>c$ in $\tilde{X}$, is the unique homotopy class to which belong the projections $p_{d}^{c}:[N(\bar{d})|\rightarrow| N(\bar{c}) \mid$. For a $c \in \tilde{X}$, let $\left[p^{c}\right]: X \rightarrow X_{c}$ be the unique homotopy class of the canonical mappings $p^{c}: X \rightarrow X_{c}$. Recall that $\left[p^{c}\right]=\left[p_{d}^{c}\right] \circ\left[p^{d}\right]$ whenever $d>c$ in $\tilde{X}$ so that $\mathbf{p}=\left\{\left[p^{c}\right]\right\}: X \rightarrow \mathcal{X}$ is a morphism of the pro-homotopy category pro-HTop. Since the usual Cech system is an $\mathcal{H}$ Pol-expansion (see [4, p. 328]), it is easy to show by direct verification of conditions (E1) and (E2), that $\mathrm{p}$ is also an $\mathcal{H} P o l$-expansion. 
In the rest of this paper, let $X, Y$, and $Z$ be topological spaces and let

$$
\begin{aligned}
& \mathbf{p}=\left\{\left[p^{a}\right]\right\}: X \rightarrow \mathcal{X}=\left\{X_{a},\left[p_{b}^{a}\right], \bar{X}\right\} \\
& \mathbf{q}=\left\{\left[q^{c}\right]\right\}: X \rightarrow \mathcal{Y}=\left\{Y_{c},\left[q_{d}^{c}\right], \tilde{Y}\right\}
\end{aligned}
$$

and

$$
\mathbf{r}=\left\{\left[r^{u}\right]\right\}: Z \rightarrow Z=\left\{Z_{u},\left[r_{v}^{u}\right], \tilde{Z}\right\}
$$

be cofinite Cech systems of $X, Y$, and $Z$, respectively.

It is well-known that shape morphisms from $X$ into $Y$ could be considered as equivalence classes of morphisms of inverse systems $\mathcal{X}$ and $\mathcal{Y}$ (see [4]). More precisely, the set $\mathcal{S h}(X, Y)$ of all shape morphisms between spaces $X$ and $Y$ can be identified with the set pro- $\mathcal{H} P$ ol $(\mathcal{X}, \mathcal{Y})$ of all morphisms in the Grothendick's pro-category pro- $\mathcal{H} P$ ol of the homotopy category of polyhedra $\mathcal{H}$ Pol between the objects $\mathcal{X}$ and $\mathcal{Y}$. In our description of what $\theta$ does on morphisms of the category $\mathcal{H M}$ we shall view shape morphisms in this way.

\section{Multi-valued functions $B_{\sigma}$.}

We shall also be using the following natural multi-valued function $B_{\sigma}$ from the nerve $|N(\sigma)|$ of a normal cover $\sigma$ of $X$ into $X$. The function $B_{\sigma}$ associates to a point $w$ of $|N(\sigma)|$ the intersection of members of the cover $\sigma$ which span a simplex of $|N(\sigma)|$ that contains $w$. Hence, if $w$ belongs to a simplex $\left\langle S_{1}, \ldots, S_{n}\right\rangle$ of $|N(\sigma)|$, where $S_{1}, \ldots, S_{n}$ are members of $\sigma$, then $B_{\sigma}(w)=\bigcap_{i=1}^{n} S_{i}$. Observe that $B_{\sigma}$ is a $\left(*_{\sigma}, \sigma\right)$-function, where $*_{\sigma}$ denotes the (normal) cover of $|N(\sigma)|$ by open stars $*_{\sigma}^{S}$ of all vertices $S \in \sigma$ of $|N(\sigma)|$. Moreover, for every canonical mapping $p: X \rightarrow|N(\sigma)|$ (i. e., a map which satisfies $p^{-1}\left(*_{\sigma}^{S}\right) \subset S$ for every member $S$ of $\sigma$ ) and every $x \in X$ there is a $V \in \sigma$ such that both $x$ and the set $B_{\sigma} \circ p(x)$ lie in $V$ while for every $x \in X$ and every $S \in \sigma$ with $x \in S$ the set $B_{\sigma} \circ p(x)$ is a subset of $\operatorname{st}(S, \sigma)$. Hence, the composition $B_{\sigma} \circ p$ is $\{\sigma, s t(\sigma))$-homotopic and $\sigma$-close to the identity map $i d_{X}$.

Lemma 3. Let $\sigma$ and $\tau$ be normal covers of a space $X$ such that $\tau$ refines $\sigma$. Then $B_{\tau} \stackrel{\sigma}{\simeq} B_{\sigma} \circ$ p for every projection $p:|N(\tau)| \rightarrow|N(\sigma)|$.

Proof: Let $T \in \tau$ and let $x \in *_{\tau}^{T}$. Then $B_{\tau}(x)$ is a subset of $T$ whilc $p(x)$ lies in $*_{\sigma}^{S}$, where $S$ is a member of $\sigma$ which contains $T$. It follows that both $B_{\tau}(x)$ and $B_{\sigma} \circ p(x)$ are subsets of $S$. Hence, the function $H$ from $|N(\tau)|$ into $X$ defined by the rule $H(x)=B_{\tau}(x) \cup B_{\sigma} \circ p(x)$ for every $x \in|N(\tau)|$ is a $\sigma$-homotopy joining $B_{\tau}$ and $B_{\sigma} \circ p$. 


\section{Approximation of small functions with maps.}

In the description of the functor $\theta$ we shall also need the following approximation result (see Lemma 2 in [2]).

Lemma 4. For every open cover $\sigma$ of a polyhedron $Y$ there is an open cover $\tau$ of it such that for every $\tau$-small multi-valued function $F: X \rightarrow$ $Y$ from a space $X$ into $Y$ there is a single-volued continuous function $f: X \rightarrow Y$ with $F \stackrel{\sigma}{=} f$.

\section{Description of the functor $\theta$}

The functor $\theta$ will leave the objects unchanged. In order to explain how $\theta$ effects the morphisms we must work much hardcr.

Let $\varphi=\left\{F_{s}\right\}_{s \in \dot{Y}}: X \rightarrow Y$ be a multi-net. For each $c \in \hat{Y}$, pick an open cover $\sigma_{c}$ of $Y_{c}=|N(\bar{c})|$ such that $s t^{3}\left(\sigma_{c}\right)$-close maps into $Y_{c}$ are homotopic. Since the set $\tilde{Y}$ is cofinite, we can select the covers $\sigma_{c}$ so that $\sigma_{d}$ refines $\left(q_{d}^{c}\right)^{-1}\left(\sigma_{c}\right)$ whenever $d>c$ in $\tilde{Y}$. Morcover, we can assume that $\sigma_{c}$ refines the open cover $*_{\bar{c}}$ for every $c \in \tilde{Y}$.

By Lemma 4 , we can find a $\tau_{c} \in \sigma_{c}{ }^{*}$ so that every $\tau_{c}$-small multi-valued function into $Y_{c}$ is $\sigma_{c}$-close to a continuous single-valued function. Let $\xi_{c} \in \hat{Y}$ be a refinement of $\left(q^{c}\right)^{-1}\left(\tau_{c}\right)$. Since $\tilde{Y}$ is a cofinite directed set, we can assume that $\xi_{c}$ refines $\xi_{e}$ for every $e \in \tilde{Y}$ with $c>e$. Moreover, for every pair $c, e \in \vec{Y}$ with $c>e$, the maps $q^{c}$ and $q_{c}^{e} \circ q^{c}$ are joined by a homotopy $K_{c}^{e}$ so that we can select a $\pi_{c}^{e} \in \hat{Y}$ and a stacked normal cover $\varrho_{c}^{e}$ of $Y \times I$ over $\pi_{c}^{e}$ which refines $\left(K_{c}^{e}\right)^{-1}\left(\tau_{e}\right)$. We shall assume that $\xi_{c}$ also refines $\pi_{c}^{e}$ for every index $e$ with $c>e$.

Since $\varphi$ is a multi-net, there is an index $\varphi_{c} \in \tilde{Y}$ so that

$$
F_{d} \stackrel{\xi_{c}}{=} F_{e} \quad \text { for all } d, e>\varphi_{c}
$$

Choose an increasing function $\varphi^{*}: \tilde{Y} \rightarrow \vec{Y}$ such that $\varphi^{*}(c)>c, \varphi_{c} ;\left\{\xi_{c}\right\}$ for every $c \in \vec{Y}$.

Since the the function $F_{\varphi^{*}(c)}$ is $\xi_{\mathrm{c}^{-}}$small, there is an $\eta_{c} \in \hat{X}$ such that $F_{\varphi^{*}(c)}$ is an $\left(\eta_{c}, \xi_{c}\right)$-function. Let $\lambda_{c} \in \eta_{i:}{ }^{*}$. Choose an increasing function $\varphi: \tilde{Y} \rightarrow \tilde{X}$ such that $\varphi(c)>\left\{\lambda_{c}\right\}$ for every $c \in \tilde{Y}$. The composition $q^{c} \circ F_{\varphi^{*}(c)} \circ B \overline{\varphi(c)}$ is a $T_{c}$-small multi-valued function so that it is $\sigma_{c}$-close to a map $\varphi^{c}: X_{\varphi(c)} \rightarrow Y_{c}$.

Claim 1. The pair $\underline{\varphi}=\left(\varphi,\left\{\varphi^{c} \mid c \in \tilde{Y}\right\}\right)$ is a morphism between cofinite Cech systems $\mathcal{X}$ and $\mathcal{Y}$. 
Proof: We must show that for every pair $c, d$ of elements of $\vec{Y}$ with $d>c$ it is possible to find an $a>x, y$ so that

$$
\varphi^{c} \circ p_{a}^{x} \simeq q_{d}^{c} \circ \varphi^{d} \circ p_{a}^{y}
$$

where $x=\varphi(c)$ and $y=\varphi(d)$.

Let $u=\varphi^{*}(c)$ and $v=\varphi^{*}(d)$. Since $v>u>\varphi_{c}$, by (1), therc is a normal cover $Q$ of $X \times I$ such that the functions $F_{u}$ and $F_{v}$ can be joined by a $\left(\varrho, \xi_{c}\right)$-homotopy $M: X \times I \rightarrow Y$. Pick a normal cover $\pi$ of $X$ and a stacked normal cover $\omega$ of $X \times I$ over $\pi$ such that $\omega$ refines $\varrho$. For $a>\{\pi\}$, we see that $Q=q^{c} \circ M \circ\left(B_{\alpha} \times i d_{I}\right)$ is a $\tau_{c}$-homotopy joining $q^{c} \circ F_{u} \circ B_{\alpha}$ and $q^{c} \circ F_{v} \circ B_{\alpha}$, where $\alpha=\bar{a}$.

Since $F_{u}$ is a $\left(\xi, \xi_{c}\right)$-function and $B_{\alpha} \stackrel{\xi}{\simeq} B_{\xi} \circ p_{a}^{x}$ by Lemma 3 , we see that there is a $\tau_{c}$-homotopy $P^{2}$ with $P_{0}=q^{c} \circ F_{u} \circ B_{\xi} \circ p_{a}^{x}$ and $P_{1}=Q_{0}$, where $\xi=\bar{x}$. For a similar reason, there is a $\tau_{c}$-homotopy $R$ with $R_{0}=Q_{1}$ and $R_{1}=q^{c} \circ F_{\nu} \circ B_{\lambda} \circ p_{a}^{y}$, where $\lambda=\bar{y}$.

Since $F_{v} \circ B_{\lambda} \circ p_{a}^{y}$ is a $\xi_{d}$-function and $\xi_{d}$ refines $\pi_{d}^{c}, S=K_{d}^{c} \circ\left(\left(F_{v} \circ B_{\lambda} \circ\right.\right.$ $\left.\left.p_{a}^{y}\right) \times i d_{I}\right)$ is a $\tau_{c}$-homotopy with $S_{0}=R_{1}$ and $S_{1}=q_{d}^{c} \circ q^{d} \circ F_{v} \circ B_{\lambda} \circ p_{a}^{y}$.

Obscrve that our choices imply the existence of homotopies $A, B, C$, and $D$ such that $A \stackrel{\sigma}{=} P, B \stackrel{\sigma}{=} Q, C \stackrel{\sigma}{=} R, D \stackrel{\sigma}{=} S, \varphi^{c} \circ p_{a}^{x}=P_{0}$, and $S_{1}=q_{d}^{c} \circ \varphi^{d} \circ p_{a}^{y}$, where $\sigma=\sigma_{c}$. From here it follows that $\varphi^{c} \circ p_{a}^{x} \stackrel{\nu}{=} A_{0}$, $A_{1} \stackrel{\nu}{=} B_{0}, B_{1} \stackrel{\nu}{=} C_{0}, C_{1} \stackrel{\nu}{=} D_{0}, D_{1} \stackrel{\nu}{=} q_{d}^{c} \circ \varphi^{d} \circ p_{a}^{y}$, where $\nu=\operatorname{st}\left(\sigma_{c}\right)$.

The way in which we selected the covers $\sigma_{c}$ implies that the adjacent maps in the following long list are homotopic: $\varphi^{c} \circ p_{a}^{x}, A_{0}, A_{1}, B_{0}, B_{1}$, $C_{0}, C_{1}, D_{0}, D_{1}, q_{d}^{c} \circ \varphi^{d} \circ p_{a}^{y}$. Hence, the relation (2) holds.

Now we can define that $\theta$ acts on morphisms of the category $\mathcal{H M}$ (i. e., on homotopy classes of multi-nets) by the rule $\theta([\varphi])=[\underline{\varphi}]$, where $[\underline{\varphi}]$ denotes the equivalence class of $\underline{\varphi}$ with respect to the equivalence relation $\sim($ see $[4$, p. 6] $)$.

Claim 2. The function $\theta$ is well-defined, $i$. e., it does not depend on the choices of $\varphi, \varphi^{*}$, and $\varphi^{C}$ in our description of $\{\underline{\varphi}\}$.

Proof: Suppose that $\psi=\left\{G_{c}\right\}: X \rightarrow Y$ is multi-net homotopic to $\varphi$ and let the morphism $\psi=\left(\psi,\left\{\psi^{c} \mid c \in \tilde{Y}\right\}\right)$ of inverse systems $\mathcal{X}$ and $\mathcal{Y}$ be constructed from $\psi$ by the above procedure using in it $\psi, \psi^{*}$, and $\psi^{c}$ instead of $\varphi, \varphi^{*}$, and $\varphi^{c}$, respectively. We must show that $\varphi$ and $\psi$ are equivalent, i. e., that for every $c \in \tilde{Y}$ there is an $a>x, u$ with

$$
\varphi^{c} \circ p_{a}^{x} \simeq \psi^{c} \circ p_{a}^{u}
$$

where $x=\varphi(c)$ and $u=\psi(c)$. 
Let a $c \in \bar{Y}$ be given. In order to prove (3), we shall argue the existence of indices $a, z \in \tilde{Y}$ such that

$$
\begin{gathered}
\varphi^{c} \circ p_{a}^{x} \stackrel{\sigma_{c}}{=} q^{c} \circ F_{y} \circ B_{\xi} \circ p_{\alpha}^{x}, \\
q^{c} \circ F_{y} \circ B_{\xi} \circ p_{a}^{x} \stackrel{\tau_{c}}{=} q^{c} \circ F_{y} \circ B_{\alpha}, \\
q^{c} \circ F_{y} \circ B_{\alpha} \stackrel{\tau_{c}}{\simeq} q^{c} \circ F_{z} \circ B_{\alpha}, \\
q^{c} \circ F_{z} \circ B_{\alpha} \stackrel{\tau_{c}}{\simeq} q^{c} \circ G_{z} \circ B_{\alpha}, \\
q^{c} \circ G_{z} \circ B_{\alpha} \stackrel{\tau_{c}}{\simeq} q^{c} \circ G_{v} \circ B_{\alpha}, \\
q^{c} \circ G_{v} \circ B_{\alpha} \stackrel{\tau_{c}}{\simeq} q^{c} \circ G_{v} \circ B_{\gamma} \circ p_{\alpha}^{u}, \\
q^{c} \circ G_{v} \circ B_{\gamma} \circ p_{\alpha}^{u} \stackrel{\sigma_{c}}{=} \psi_{c} \circ p_{\alpha}^{u},
\end{gathered}
$$

where $\xi=\bar{x}, y=\varphi^{*}(c), \gamma=\bar{u}, v=\psi^{*}(c)$, and $\alpha=\bar{a}$.

Once we have the relations (4)-(10), we approximate each of the $\tau_{c^{-}}$ homotopies by a $\sigma_{c}$-close homotopy and conclude from here as in the proof of Claim 1 that (3) holds.

Add (4) and (10). This follows from the fact that $\varphi^{c}$ and $\psi^{c}$ are $\sigma_{c}$-close to functions $q^{c} \circ F_{y} \circ B_{\xi}$ and $q^{c} \circ G_{v} \circ B_{\gamma}$, respectively.

Add (5). It suffices to observe that $F_{y}$ is a $\left(\xi, \xi_{c}\right)$-function and that $B_{\alpha} \stackrel{\xi}{\simeq} B_{\xi} \circ p_{a}^{x}$ for every $a>x$.

Add (6). Let $z>y$. Then there is a normal cover $\lambda$ of $X \times I$ and a $\left(\lambda, \xi_{c}\right)$-homotopy $L$ joining $F_{y}$ and $F_{z}$. Pick a normal cover $\pi$ of $X$ and a stacked normal cover $\varrho$ of $X \times I$ over $\pi$ such that $\varrho$ refines $\lambda$. Let $a>\{\pi\}$. The composition $q^{c} \circ L \circ\left(B_{\alpha} \times i d_{I}\right)$ is a $\tau_{c}$-homotopy which realizes the relation (6).

Add (7). Since $\varphi$ and $\psi$ are homotopic multi-nets, there is a $b \in \tilde{Y}$ such that $F_{z} \stackrel{\xi_{c}}{\simeq} G_{z}$ for every $z>b$. Let $z>b$ and let $M$ be a $\xi_{c^{-}}$ homotopy joining $F_{z}$ and $G_{z}$. Choose normal covers $\lambda$ and $\varrho$ of $X \times I$ and $\pi$ of $X$ such that $M$ is a $\left(\lambda, \xi_{c}\right)$-function, $\varrho$ refines $\lambda$ and $\varrho$ is stacked over $\pi$. Let $a>\{\pi\}$. Then the composition $q^{c} \circ M \circ\left(B_{\alpha} \times i d_{I}\right)$ is a $\tau_{c}$-homotopy which realizes the relation (7).

Add (8) and (9). These are analogous to relations (6) and (5), respectively. 
Claim 3. Let $\iota=\left\{I_{a}\right\}_{a \in \bar{X}}$ be the identity multi-net on a space $X$, where $I_{\alpha}=i d_{X}$ for every $a \in \tilde{X}$. Then the homotopy class [ $\underline{\underline{l}}$ associated to the homotopy class [l] by the function $\theta$ is the identity homotopy class $\left[\left(i d_{\bar{X}},\left\{I_{a} \mid a \in \bar{X}\right\}\right)\right]$.

Proof: We must show that for every $a \in \tilde{X}$ there is $a b>a, \iota(a)$ such that

$$
p_{b}^{a} \simeq \iota^{a} \circ p_{b}^{l(a)} .
$$

In order to prove the above statement, observe that we can assume that the function $\iota: \tilde{X} \rightarrow \tilde{X}$ (which corresponds to the function $\varphi$ in the above description) has the property that the cover $\xi$ is a star-refinement of the cover $\left(p^{a}\right)^{-1}\left(\tau_{a}\right)$ for every $a \in \tilde{X}$, where $\xi=\bar{x}$ and $x=\iota(a)$.

Let an $a \in \tilde{X}$ be given. By construction, we have $\iota^{a} \circ p^{x} \stackrel{\sigma_{a}}{=}$ $p^{\alpha} \circ B_{\xi} \circ p^{x}$.

Since $B_{\xi} \circ p^{x}$ is $s t(\xi)$-homotopic to the identity map $i d_{X}$ on $X$ and $s t(\xi)$ refines $\left(p^{a}\right)^{-1}\left(\tau_{a}\right)$, there is a $\tau_{a}$-homotopy $H$ with $H_{0}=p^{a} \circ B_{\xi} \circ p^{x}$ and $H_{1}=p^{a}$.

Let $K$ be a single-valued continuous function $\sigma_{a}$-close to $H$. Then $\iota^{a} \circ p^{x}$ is $s t\left(\sigma_{a}\right)$-close to $K_{0}$ and $K_{1}$ is $\sigma_{a}$-close to $p^{a}$. It follows that $\iota^{a} \circ p^{x}$ and $p^{a}$ are homotopic. Finally, we can use the property (E2) of the cofinite Cech system $\mathcal{X}$ to get a $b>a, \iota(a)$ such that (11) holds.

Claim 4. For multi-nets $\varphi=\left\{F_{c}\right\}_{c \in \bar{Y}}: X \rightarrow Y$ and $\psi=\left\{G_{u}\right\}_{u \in \bar{Z}}:$ $Y \rightarrow Z$,

$$
\theta([\psi] \circ[\varphi])=\theta([\psi]) \circ \theta([\varphi]) \text {. }
$$

Proof: Let $\chi=\left\{H_{u}\right\}: X \rightarrow Z$, where $H_{u}=G_{g(u)} \circ F_{f\left(\left\{g^{-}(u)\right\}\right)}$ for every $u \in \bar{Z}$. Then $[\chi]=[\psi] \circ[\varphi]$. Let $\underline{\varphi}=\left(\varphi,\left\{\varphi^{c}\right\}_{\mathrm{c} \in \bar{Y}}\right)$, $\psi=\left(\psi,\left\{\psi^{u}\right\}_{u \in \bar{Z}}\right)$, and $\underline{\chi}=\left\langle\chi,\left\{\chi^{u}\right\}_{u \in \bar{Z}}\right)$ be obtained from $\varphi, \psi$, and $\chi$ by the above procedure. We must show that $\underline{\chi}$ and $\underline{\psi} \circ \underline{\varphi}$ are homotopic. Since $\underline{\psi} \circ \underline{\varphi}=\left(\varphi \circ \psi,\left\{\psi^{u} \circ \varphi^{\psi(u)}\right\}_{u \in \bar{Z}}\right)$, this amounts to show that for every $\bar{u} \in \overline{\bar{Z}}$ there is an $a>t, x$ such that

$$
\chi^{u} \circ p_{a}^{b} \simeq \psi^{u} \circ \varphi^{k} \circ p_{a}^{t},
$$

where $t=\varphi \circ \psi(u), k=\psi(u)$, and $b=\chi(u)$.

Let a $u \in \tilde{Z}$ be given. In order to prove the above statement, we shall argue the existence of large enough indices $w \in \bar{Z}, z \in \tilde{Y}$, and $a \in \tilde{X}$ such that

$$
\chi^{u} \circ p_{a}^{b} \stackrel{\alpha_{u}}{=} r^{u} \circ H_{x} \circ B_{\beta} \circ p_{a}^{b}
$$




$$
\begin{gathered}
r^{u} \circ H_{x} \circ B_{\beta} \circ p_{a}^{b} \stackrel{\beta_{u}}{\simeq} r^{u} \circ H_{x} \circ B_{\alpha}, \\
r^{u} \circ H_{x} \circ B_{\alpha} \stackrel{\beta_{u}}{\simeq} r^{u} \circ G_{y} \circ F_{z} \circ B_{\alpha}, \\
r^{u} \circ G_{y} \circ F_{z} \circ B_{\alpha} \stackrel{\beta_{u}}{\simeq} r^{u} \circ G_{w} \circ F_{z} \circ B_{\alpha}, \\
r^{u} \circ G_{w} \circ F_{z} \circ B_{\alpha} \stackrel{\beta_{u}}{\simeq} r^{u} \circ G_{e} \circ F_{z} \circ B_{\alpha}, \\
r^{u} \circ G_{e} \circ F_{z} \circ B_{\alpha} \stackrel{\beta_{u}}{\simeq} r^{u} \circ G_{e} \circ F_{i} \circ B_{\alpha}, \\
r^{u} \circ G_{e} \circ F_{i} \circ B_{\alpha} \stackrel{\beta_{u}}{\simeq} r^{u} \circ G_{e} \circ F_{\imath} \circ B_{\tau} \circ p_{a}^{t}, \\
r^{u} \circ G_{e} \circ F_{i} \circ B_{\tau} \circ p_{o}^{t} \stackrel{\beta_{u}}{\simeq} r^{u} \circ G_{e} \circ B_{\kappa} \circ q^{k} \circ F_{i} \circ B_{\tau} \circ p_{a}^{t}, \\
r^{u} \circ G_{e} \circ B_{\kappa} \circ q^{k} \circ F_{i} \circ B_{\tau} \circ p_{a}^{\ell} \stackrel{\alpha_{u}}{=} r^{u} \circ G_{e} \circ B_{\kappa} \circ \varphi^{k},
\end{gathered}
$$

and

$$
r^{u} \circ G_{e} \circ B_{\kappa} \circ \varphi^{k} \stackrel{\alpha_{u}}{=} \psi^{u} \circ \varphi^{k} \circ p_{a}^{t},
$$

where $\alpha=\bar{a}, x=\chi^{*}(u), \beta=\bar{b}, y=\psi(u), e=\psi^{*}(u), i=\varphi^{*}(k), \tau=\bar{t}$, $\kappa=\bar{k}$, and $\alpha_{u}$ and $\beta_{u}$ are covers of $Z_{u}$ analogous to covers $\sigma_{c}$ and $\tau_{c}$ of $Y_{c}$, respectively.

Once we have the relations (13)-(22), we approximate each of the $\beta_{u^{*}}$ homotopies by an $\alpha_{z}$-close homotopy and conclude from here as in the proof of Claim 1 that (12) holds.

Add (13) and (22). This follows from the fact that $\chi^{u}$ and $\psi \psi^{u}$ are $\alpha_{u}$-close to functions $r^{u} \circ H_{x} \circ B_{\beta}$ and $r^{u} \circ G_{e} \circ B_{\kappa}$, respectively.

Add (21). Observe that $r^{u} \circ G_{e}$ is a $\left(\kappa, \alpha_{u}\right)$-function, $B_{\kappa}$ is a $\left(*_{k}, \kappa\right)$ function, the cover $\sigma_{k}$ refines the cover $*_{k}$, and the map $\varphi^{k}$ is $\sigma_{k}$-close to the composition $q^{k} \circ F_{i} \circ B_{\tau}$, where $*_{k}$ denotes the normal cover of $Y_{k}$ by the open stars of all vertices.

Add (14). Notice that $r^{2 t} \circ H_{x}$ is a $\left(\beta, \beta_{u}\right)$-function and $B_{\alpha} \stackrel{\beta}{\simeq} B_{\beta} \circ p_{a}^{b}$ for every $a>b$. 
Add (15). Recall that $H_{x}=G_{y} \circ F_{s}$, where $s=f\left(\left\{g^{*}(x)\right\}\right)$. Since $r^{u} \circ G_{u}$ is a $\left(g^{*}(x), \beta_{u}\right)$-function, it suffices to take $z>s$ because then $F_{s}$ and $F_{z}$ are joined by the $g^{*}(u)$-homotopy $L$ so that the composition $r^{u} \circ G_{y} \circ L \circ\left(B_{\alpha} \times i d_{I}\right)$ will be a $\beta_{u}$-homotopy which realizes (15) whenever $a$ is sufficiently large.

Add (16). Let $w>y$. Then $G_{y}$ and $G_{w}$ are joined by a $\xi$-homotopy $M$, where $\xi=\bar{x}$. But, the cover $\xi$ refines the cover $\gamma_{u}=\left(r^{u}\right)^{-1}\left(\beta_{u}\right)$. It follows that $r^{u} \circ M$ is a $\beta_{u}$-homotopy joining $r^{u} \circ G_{y}$ and $r^{u} \circ G_{w}$. Since for every normal cover $\lambda$ of $Y$ we can find indices $a$ and $z$ such that the composition $F_{z} \circ B_{\alpha}$ is a $\lambda$-small function, it is clear that there arc indices $z$ and $a$ such that $r^{u} \circ M \circ\left(F_{z} \circ B_{\alpha} \times i d_{l}\right)$ realizes the relation (16).

Add (17). Let $w>e$. Then $G_{w}$ and $G_{e}$ are joined by a $\gamma_{u}$-small homotopy $N$. As in the proof of (16), we get the existence of large enough indices $z$ and $a$ such that $r^{u} \circ N \circ\left(F_{z} \circ B_{\alpha} \times i d_{I}\right)$ realizes the relation (17).

Add (18). Observe that $G_{e}$ is a $\left(\kappa, \gamma_{u}\right)$-function. Also, we can always assume that the cover $\xi_{k}$ was selected so that it refines the cover $\kappa$. Then for $z>i$ the functions $F_{z}$ and $F_{i}$ are joined by the $\xi_{k}$-homotopy $P$. Hence, for a large index $a$, the composition $r^{u} \circ G_{e} \circ P^{\circ} \circ\left(B_{\alpha} \times i d_{l}\right)$ realizes the relation (18).

Add (19). The argument for this relation is similar to the one given for the relation (14).

Add (20). Notice that $r^{u} \circ G_{e}$ is a $\left(\mu, \gamma_{u}\right)$-function, where $\mu$ is a normal cover of $Y$ such that $s t(\kappa)$ refines $\mu$. Also, there is a $\left(\kappa, s t(\kappa)\right.$ )-homotopy $R$ joining $i d_{X}$ with the composition $B_{\kappa} \circ q^{k}$. Once again, if we choose the cover $\xi_{k}$ so that it refines the cover $\kappa$, then $r^{u} \circ G_{e} \circ R \circ\left(F_{i} \circ B_{\tau} \circ p_{a}^{t} \times i d_{I}\right)$ will be a $\beta_{u}$-homotopy that realizes the relation $(20)$.

Claim 5. $\theta$ is a functor and the relation $S=\theta \circ J$ holds.

Proof: That $\theta$ is a functor follows from the Claims 3 and 4 . It remains to see that $S=0 \circ J$. Let $f: X \rightarrow Y$ be a map, i. e., a morphism of the category Top. Then $J(f)$ is represented by a multi-net $\varphi=\left\{F_{c}\right\}_{c \in \dot{Y}}$ : $X \rightarrow Y$, where $F_{c}=f$ for every $c \in \tilde{Y}$. It follows that $\theta \circ J(f)$ is represented by a morphism $\varphi=\left(\varphi,\left\{\varphi^{c}\right\}_{c \in \dot{Y}}\right)$ between inverse systems $\mathcal{X}$ and $\mathcal{Y}$, where $\varphi^{c}$ is a map which is $\sigma_{c}$-close to $q^{c} \circ f \circ B_{\alpha}$ for a suitable index $a \in \tilde{X}$ and the cover $\alpha=\bar{a}$ of $X$. We shall now prove that

$$
\varphi^{c} \circ p^{a} \simeq q^{c} \circ f \text {. }
$$

Observe that

$$
\varphi^{c} \circ p^{a} \stackrel{\sigma_{c}}{=} q^{c} \circ f \circ B_{\alpha} \circ p^{a} .
$$


But, $B_{\alpha} \circ p^{\alpha}$ is $\alpha$-close to $i d_{X}$. Hence, had we chosen $a$ so that $\alpha$ refines the cover $\left(q^{c} \circ f\right)^{-1}\left(\sigma_{c}\right)$, we would get

$$
q^{c} \circ f \circ B_{\alpha} \circ p^{a} \stackrel{\sigma_{c}}{=} q^{c} \circ f \text {. }
$$

From (24) and (25) it follows that $\varphi^{c} \circ p^{a}$ and $q^{c} \circ f$ are $s t\left(\sigma_{c}\right)$-close and we get the relation (23).

In order to conclude now that $S(f)=\theta \circ J(f)$ we must recall (see [4]) that $S(f)$ is a unique morphism $\underline{f}$ such that $q \circ f=\underline{f} \circ \mathbf{p}$.

We shall now prove that $\theta$ is a category isomorphism by constructing for every pair of objects $X$ and $Y$ of the shape category a function

$$
\zeta: \operatorname{Mor}_{S h}(X, Y) \rightarrow \operatorname{Mor}_{\mathcal{H} M}(X, Y)
$$

such that $\theta \circ \zeta=i d$ and $\zeta \circ \theta=i d$.

\section{Preliminaries for the description of the functor $\zeta$}

\section{Factorization through canonical mappings.}

Lemma 5. Let $X$ be an arbitrary space, let $Y$ be a polyhedron (endowed with the $C W$ topology), let $\sigma$ be an open covering of $Y$, and let $f: X \rightarrow Y$ be a map. Then there exist a normal cover $\tau$ of $X$ such that for every normal cover $\varrho$ of $X$ which refines $\tau$ there exist a map $k:|N(\varrho)| \rightarrow Y$ with the following properties:

(i) For any canonical map $p: X \rightarrow|N(\varrho)|$ the maps $k \circ p$ and $f$ are $\sigma$-close.

(ii) Each $R \in \varrho$ admits an $S_{R} \in \sigma$ such that

(a) $k\left(*_{e}^{R}\right) \subset S_{R}$, and

(b) $f(R) \subset S_{R}$.

Proof: Let $\delta \in \sigma^{*}$. By [4], there is an ANR space $\mathrm{M}$ and maps $u$ : $Y \rightarrow M$ and $d: M \rightarrow Y$ such that

$$
d \circ u \stackrel{\delta}{=} i d_{Y} \text {. }
$$

Let $\beta=d^{-1}(\delta)$. Let $h=u \circ f$. By Lemma 2 on p. 316 of [4], there is a normal cover $\tau$ of $X$ and a map $g:|N(\tau)| \rightarrow M$ with the following properties:

(i') For any canonical map $r: X \rightarrow|N(\tau)|$ the maps $g \circ r$ and $h$ are $\beta$-close.

(ii') Each $T \in \tau$ admits a $B_{T} \in \beta$ such that

(a') $g\left(*_{T}^{T}\right) \subset S_{T}$, and

(b) $h(T) \subset S_{T}$. 
Let $\varrho \in \hat{X}$ be a refinement of $\tau$ and let $q:|N(\varrho)| \rightarrow|N(\tau)|$ be a simplicial projection mapping induced by the selection of a member $\nu(R)$ of $\tau$ with $R \subset \nu(R)$ for every member $R$ of $\varrho$. Let $k=d \circ g \circ q$.

In order to check (i), let $p: X \rightarrow|N(\varrho)|$ be a canonical map. By Theorem 6 on $p$. 326 of $[4]$, the composition $r=q \circ p$ is also a canonical map. By (i'), the maps $g \circ r$ and $h$ are $\beta$-close. Composing with the map $d$ we see that $k \circ p$ and $d \circ u \circ f$ are $\delta$-close. But, by (26), $d \circ u \circ f$ is $\delta$-close to $f$ so that $f \stackrel{g}{=} k \circ p$.

Finally, to verify (ii), let $R \in \varrho$. By (ii'), there is a $D_{\nu(R)} \in \delta$ such that

(c) $g\left(*_{\tau}^{\nu(R)}\right) \subset d^{-1}\left(D_{\nu(R)}\right)$, and

(d') $h\left(\nu(R) \subset d^{-1}\left(D_{\nu(R)}\right)\right.$.

For each $R \in \varrho$ choose an $S_{R} \in \sigma$ such that $S_{R}$ contains the star $\operatorname{st}\left(D_{\nu(R)}, \delta\right)$ of $D_{\nu(R)}$ with respect to the cover $\delta$. Then

$$
k\left(*_{\varrho}^{R}\right)=d \circ g \circ q\left(*_{\varrho}^{R}\right) \subset d \circ g\left(*_{\tau}^{\nu(R)}\right) \subset D_{\nu(R)} \subset S_{R} .
$$

On the other hand, from (d') we get $d \circ u \circ f(R) \subset D_{\nu(R)}$ for every $R \in \varrho$. But, by (26), for each $y \in f(R)$, some member of $\delta$ contains both $y$ and $d \circ u(y)$. Hence, $f(R) \subset S_{R}$.

\section{Hooked and small implies homotopic.}

The following notion and lemma are from [2]. Let $\sigma$ be a normal cover of a space $Y$. Two multi-valued functions $F, G: X \rightarrow Y$ are $\sigma$-hooked provided for every $x \in X$ there is an $S_{x} \in \sigma$ such that $S_{x}$ has non-empty intersection with both $F(x)$ and $G(x)$. Observe that $\sigma$-close multi-valued functions are $\sigma$-hooked.

Lemma 6. Let $F, G: X \rightarrow Y$ be multi-valued functions and let $\sigma$ be a normal cover of $Y$. If $F$ and $G$ are $\sigma$-small and $\sigma$-hooked, then $F \stackrel{s t(\sigma)}{\simeq} G$.

\section{Description of the functor $\zeta$}

Let $\underline{f}=\left(f,\left\{f^{c}\right\}_{c \in \bar{Y}}\right)$ be a morphism between cofinite Čech systems $\mathcal{X}$ and $\mathcal{Y}$ associated to spaces $X$ and $Y$, respectively. For every $c \in \tilde{Y}$, define a multi-valued function $f_{c}: X \rightarrow Y$ to be the composition $B_{\gamma} \circ f^{c} \circ p^{x}$, where $\gamma$ denotes the normal cover $\bar{c}$ and $x=f(c)$. Let $\bar{f}=\left\{f_{c}\right\}_{c \in \dot{Y}}$. 
Claim 6. The family $\bar{f}$ is a multi-net from $X$ into $Y$.

Proof: Let a $\sigma \in \hat{Y}$ be given. We must find an index $c \in \tilde{Y}$ such that

$$
f_{c} \stackrel{\sigma}{\simeq} f_{d} \quad \text { for every } d>c .
$$

Let $\tau \in \sigma^{* 2}$. Put $c=\{\tau\}$. Let $d>c$. Since $f$ is a morphism between $\mathcal{X}$ and $\mathcal{Y}$, there is an index $a>x, y$ and a homotopy $H$ with

$$
H_{0}=f^{c} \circ p_{a}^{x} \text { and } H_{1}=q_{d}^{c} \circ f^{d} \circ p_{a}^{y} \text {; }
$$

where $x=f(c)$ and $y=f(d)$. Morcover, there are homotopies $G$ and $K$ with

$$
G_{0}=p^{x}, \quad G_{1}=p_{a}^{x} \circ p^{a}, \quad K_{0}=p_{a}^{y} \circ p^{a}, \quad K_{1}=p^{y} .
$$

Let $\gamma=\bar{c}$ and $\delta=\bar{d}$. Let $L$ be a $\tau$-homotopy joining $B_{\gamma} \circ q_{d}^{c}$ and $B_{\delta}$ (for this see Lemma 3). The compositions $A=B_{\gamma_{j}} \circ f^{c} \circ G, C=$ $L \circ\left(f^{d} \circ p_{a}^{y} \circ p^{a} \times i d_{J}\right), B=B_{\gamma} \circ H \circ\left(p^{a} \times i d_{l}\right)$, and $D=B_{\delta} \circ f^{d} \circ K$ are $\tau$-homotopies such that $f_{c}=A_{0}, A_{1}=B_{0}, B_{1}=C_{0}, C_{1}=D_{0}$, and $D_{1}=f_{d}$. Hence, $f_{c} \stackrel{\sigma}{\simeq} f_{d}$.

Now we can define the function $\zeta$ by the rule $\zeta([\underline{f}])=[\ddot{f}]$.

Claim 7. The function $\zeta$ is well-defined, i. e., the volue $\zeta([f])$ does not depend on the choice of the representative $f$ of the equivalence class [f].

Proof: Let $g=\left(g,\left\{g^{c}\right\}_{c \in \bar{Y}}\right)$ be another morphism from $\mathcal{X}$ into $\mathcal{Y}$ equivalent to $\bar{f}$ and let $\bar{g}=\left\{g_{c}\right\}_{c \in \bar{Y}}$ be a multi-net constructed from $\underline{g}$ by the above procedure. Wo must show that $\bar{f}$ and $\bar{g}$ are homotopic, $\mathrm{i}$. e., that for every $\sigma \in \hat{Y}$ there is an index $c \in \tilde{Y}$ such that

$$
f_{d} \stackrel{\sigma}{\simeq} g_{d} \quad \text { for every } d>c \text {. }
$$

Let a $\sigma \in \hat{Y}$ be given. Let $\tau \in \sigma^{*}$. Put $c=\{\tau\} \in \dot{Y}$. Let $d>c$. Since morphisms $\underline{f}$ and $\underline{g}$ are equivalent, there is an index $a>x, y$ and a homotopy $H$ with

$$
H_{0}=f^{d} \circ p_{a}^{x} \text { and } H_{1}=g^{d} \circ p_{a}^{y} .
$$

Moreover, there are homotopies $G$ and $K$ with

$$
G_{0}=p^{x} ; \quad G_{1}=p_{a}^{x} \circ p^{a}, \quad K_{0}=p_{a}^{y} \circ p^{a} \quad \text { and } \quad K_{1}=p^{y},
$$

where $x=f(d)$ and $y=g(d)$. Let $\delta=\bar{d}$.

The compositions $A=B_{\delta} \circ f^{d} \circ G, B=B_{\delta} \circ H \circ\left(p^{a} \times i d_{I}\right)$, and $C=$ $B_{\delta} \circ g^{d} \circ K$ are $\tau$-homotopies such that $f_{d}=A_{0}, A_{1}=B_{0}, B_{1}=C_{0}$, and $C_{1}=g_{d}$. Hence, $f_{d} \stackrel{\sigma}{\simeq} g_{d}$. 


\section{Verification that $\zeta$ is an inverse of $\theta$}

Claim 8. For every morphism $\underline{f}=\left(f,\left\{f^{c}\right\}_{c \in \dot{Y}}\right): \mathcal{X} \rightarrow \mathcal{Y}$ we hove $[\underline{f]}=\theta \circ \zeta([\underline{f}])$.

Proof: Let $\zeta([f])=[\varphi]$, where $\varphi=\left\{f_{c}\right\}_{c \in \bar{Y}}: f_{c}=B_{\gamma} \circ f^{c} \circ p^{x}, \gamma=\bar{c}$, and $x=f(c)$ for every $c \in \vec{Y}$. Let

$$
\theta(\zeta([\underline{f}]))=\theta([\varphi])=\left[\left(\varphi,\left\{\varphi^{c}\right\}_{c \in \dot{Y}}\right)\right],
$$

where $\varphi^{c}$ is a map which is $\sigma_{c}$-close to $q^{c} \circ f_{b} \circ B_{\varepsilon}, b=\varphi^{*}(c), e=\varphi(c)$, and $\varepsilon=\bar{e}$. Hence, $\varphi^{c}$ is a map which is $\sigma_{c}$-close to $q^{c} \circ B_{\beta} \circ f^{b} \circ p^{d} \circ B_{\varepsilon}$, where $\beta=\bar{b}$ and $d=f(b)$.

Let us apply Lemma 5 in the case $X=Y, M=Y_{c}, \sigma=\tau_{c}$, and $h=q^{c}$ to get a cover $\mu_{c}$ of $Y$ such that for every refinement $\varrho$ of $\mu_{c}$ there exist a map $g_{\ell}^{c}:|N(\varrho)| \rightarrow Y_{c}$ with the following properties:

(32) For any canonical map $p: Y \rightarrow|N(\varrho)|$ the maps $g_{\varrho}^{c} \circ p$ and $q^{c}$ are $\tau_{c}$-close.

(33) Each $R \in \varrho$ admits a $T_{R} \in \tau_{C}$ such that $g_{\varrho}^{c}\left(*_{e}^{R}\right) \subset T_{R}$ and $q^{c}(R) \subset T_{R}$.

Without loss of generality, we can assume that the function $\varphi^{*}$ also satisfies the condition $\varphi^{*}(c)>\left\{\mu_{c}\right\}$ for every $c \in \tilde{Y}$. By assumption, there is a map $g_{b}^{c}: Y_{b} \rightarrow Y_{c}$ such that

$$
q^{c} \stackrel{\tau_{c}}{=} g_{b}^{c} \circ q^{b}
$$

and each $R \in \beta$ admits a $T_{R} \in \tau_{c}$ such that $g_{b}^{c}\left(*_{\beta}^{R}\right) \subset T_{R}$ and $q^{c}(R) \subset T_{R}$. But, the function $B_{\beta}$ satisfies $B_{\beta}\left(*_{\beta}^{R}\right) \subset R$ for every $R \in \beta$ so that both $g_{b}^{c}\left(*_{\beta}^{R}\right)$ and $q^{c} \circ B_{\beta}\left(*_{\beta}^{R}\right)$ are contained in $T_{R}$ for every $R \in \beta$. It follows that $g_{b}^{c}$ and $q^{c} \circ B_{\beta}$ are $\left(*_{\beta}, \tau_{c}\right)$-close. This means that by selecting the function $\varphi$ carefully, we can achieve that the map $B_{\varepsilon}$ is small enough so that

$$
g_{b}^{c} \circ f^{b} \circ p^{d} \circ B_{\varepsilon} \stackrel{T_{c}}{=} q^{c} \circ B_{\beta} \circ f^{b} \circ p^{d} \circ B_{\varepsilon} .
$$

Now we can apply Lemma 5 again, this time in the case $X=X$, $M=X_{d}, \sigma=\kappa_{c}$, where $\kappa_{c}=\left(g_{b}^{c} \circ f^{b}\right)^{-1}\left(\tau_{c}\right)$, and $h=p^{d}$ to get a cover $\nu_{c}$ of $X$ such that for every refinement $\varrho$ of $\nu_{c}$ there exist a map $h_{e}^{d}:|N(\varrho)| \rightarrow X_{d}$ with the following propertics:

(36) For any canonical map $p: X \rightarrow|N(\varrho)|$ the maps $h_{\varrho}^{d} \circ p$ and $p^{d}$ are $\kappa_{c}$-close.

(37) Each $R \in \varrho$ admits a $K_{R} \in \kappa_{c}$ such that $h_{\varrho}^{d}\left(*_{\varrho}^{R}\right) \subset K_{R}$ and $p^{d}(R) \subset K_{R}$. 
Without loss of generality, we can assume that the function $\varphi$ also satisfies the condition $\varphi(c)>\left\{\nu_{c}\right\}$ for every $c \in \tilde{Y}$. Recall that $e=\varphi(c)$. By assumption, there is a map $h_{e}^{d}: X_{e} \rightarrow X_{d}$ such that

$$
p^{d} \stackrel{\kappa_{c}}{=} h_{e}^{d} \circ p^{e}
$$

and each $E \in \varepsilon$ admits a $K_{E} \in \kappa_{c}$ such that $h_{e}^{d}\left(*_{\varepsilon}^{E}\right) \subset K_{E}$ and $p^{d}(E) \subset K_{E}$. But, the function $B_{\varepsilon}$ satisfies $B_{\varepsilon}\left(*_{\varepsilon}^{E}\right) \subset E$ for every $E \in \varepsilon$ so that both $h_{e}^{d}\left(*_{\varepsilon}^{E}\right)$ and $p^{d} \circ B_{\varepsilon}\left(*_{\varepsilon}^{E}\right)$ are subsets of $K_{E}$ for every $E \in \varepsilon$. It follows that $h_{e}^{d}$ and $p^{d} \circ B_{\varepsilon}$ are $\kappa_{c}$-close. Hence,

$$
g_{b}^{c} \circ f^{b} \circ p^{d} \circ B_{\varepsilon} \stackrel{\tau_{c}}{=} g_{b}^{c} \circ f^{b} \circ h_{e}^{d} .
$$

Since $\tau_{c} \in \sigma_{c}^{*}$, the relations (35) and (39) give that the map $g_{b}^{c} \circ f^{b} \circ h_{e}^{d}$ is $\sigma_{c}$-close to the composition $q^{c} \circ B_{\beta} \circ f^{b} \circ p^{d} \circ B_{\varepsilon}$. Therefore, we can take $g_{b}^{c} \circ f^{b} \circ h_{e}^{d}$ as the map $\varphi^{c}$.

It remains to see that the morphisms $\underline{f}$ and $\left(\varphi,\left\{\varphi^{c}\right\}_{c \in \tilde{Y}}\right)$ are equivalent, i. e., that for every $c \in \tilde{Y}$ there is an $a>e, x$ such that

$$
f^{c} \circ p_{a}^{x} \simeq g_{b}^{c} \circ f^{b} \circ h_{e}^{d} \circ p_{a}^{e} .
$$

The relations (38) and (34) imply

$$
g_{b}^{c} \circ f^{b} \circ h_{e}^{d} \circ p^{e} \simeq g_{b}^{c} \circ f^{b} \circ p^{d},
$$

and

$$
q^{c} \simeq g_{b}^{c} \circ q^{b} .
$$

From the relation (42) and the property (E2) for the expansion $q$, we get the existence of an index $i>b, c$ with

$$
q_{i}^{c} \simeq g_{b}^{c} \circ q_{i}^{b} .
$$

On the other hand, since $\underline{f}$ is a morphism, we have

$$
f^{b} \circ p^{d} \simeq q_{i}^{b} \circ f^{i} \circ p^{y}
$$

where $y=f(i)$. Thus,

$$
g_{b}^{c} \circ f^{b} \circ p^{d} \simeq g_{b}^{c} \circ q_{i}^{b} \circ f^{i} \circ p^{y} \simeq q_{i}^{c} \circ f^{i} \circ p^{y} \simeq f^{c} \circ p^{x} .
$$

It follows from (41) and (45) that $f^{c} \circ p^{x} \simeq g_{b}^{c} \circ f^{b} \circ h_{e}^{d} \circ p^{e}$. Now, we can use the property (E2) for the expansion $p$ to get an index a such that (40) holds. 
Claim 9. For every multi-net $\varphi=\left\{F_{s}\right\}_{s \in \bar{Y}}: X \rightarrow Y$ we have $\zeta \circ \theta(\mid \varphi])=[\varphi]$.

Proof: Let $\theta([\varphi])=\left[\left(\varphi,\left\{\varphi^{c}\right\}_{c \in \bar{Y}}\right)\right]$, where $\varphi^{c}: X_{\varphi(\mathrm{c})} \rightarrow Y_{c}$ is a map such that

$$
\varphi^{c} \stackrel{\sigma_{c}}{=} q^{c} \circ F_{\varphi^{*}(c)} \circ B_{\overline{\varphi(c)}} \quad \text { for every } c \in \dot{Y} .
$$

Then $\zeta \circ \theta([\varphi])=[\bar{\varphi}]$, where $\bar{\varphi}=\left\{\varphi_{c}\right\}_{c \in \bar{Y}}$ is a multi-net from $X$ into $Y$ and $\varphi_{c}=B_{\bar{c}} \circ \varphi^{c} \circ p^{\varphi(c)}$ for every $c \in \tilde{Y}$.

We must show that multi-nets $\varphi$ and $\bar{\varphi}$ are homotopic, i. e., that for every $\sigma \in \hat{Y}$ there is a $c \in \hat{Y}$ such that

$$
F_{d} \stackrel{\sigma}{\simeq} \varphi_{d} \quad \text { for every } d>c .
$$

Let a $\sigma \in \hat{Y}$ be given. Let $\tau \in \sigma^{* 3}$. Since $\varphi$ is a multi-net there is a $c>\{\tau\}$ such that

$$
F_{e} \stackrel{\tau}{\simeq} F_{d} \quad \text { for all } e, d>c .
$$

Let $d>c$. Let $\delta=\bar{d}$. Since $B_{\delta}$ is a $\left(*_{\delta}, \delta\right)$-function and $\sigma_{d}$ refines $*_{\delta}$, from (46) we get

$$
\varphi_{d} \stackrel{\tau}{=} B_{\delta} \circ q^{d} \circ F_{m} \circ B_{\nu} \circ p^{n},
$$

where $m=\varphi^{*}(d), n=\varphi(d)$, and $\nu=\bar{n}$. Also, $\varphi_{d}$ is a $\tau$-small function while the right hand side of (49) is a $\tau$-small function provided we make sure that the functions $\varphi^{*}$ and $\varphi$ increase sufficiently fast (so that $m>c$ and $\nu$ is such that $F_{m}$ is a $(\nu, \tau)$-function). It follows from Lemma 6 that

$$
\varphi_{d} \stackrel{s t(\tau)}{\simeq} B_{\delta} \circ q^{d} \circ F_{m} \circ B_{\nu} \circ p^{n}
$$

Since the composition $B_{\delta} \circ q^{d}$ is $(\delta, s t(\delta))$-homotopic to the $i d_{X}$, we see again that by a careful selection of functions $\varphi^{*}$ and $\varphi$, we can achieve that

$$
B_{\delta} \circ q^{d} \circ F_{m} \circ B_{\nu} \circ p^{n} \stackrel{s t(\tau)}{\simeq} F_{m} \circ B_{\nu} \circ p^{n} .
$$

Since $m>c$, there is a normal cover $\lambda$ of $X$ such that $F_{m}$ is a $(\lambda, \tau)$ function. Hence, if we require in addition that $\operatorname{st}(\nu)$ refines $\lambda$, then

$$
F_{m} \circ B_{\nu} \circ p^{n} \stackrel{\tau}{\simeq} F_{m}
$$

because $B_{\nu} \circ p^{n}$ is $s t(\nu)$-homotopic to the $i d_{X}$.

Finally, since $m, d>c$ from (48) we get

$$
F_{m} \stackrel{\tau}{\simeq} F_{d} .
$$

The relations (50)-(53) together imply the relation (47). 


\section{References}

1. R. A. Aló and M. L. Shapiro, "Normal Topologicol Spaces," Cambridge Univ. Rress, Cambridge, 1972.

2. Z. CERIN, Shape via multi-nets, preprint.

3. A. Dold, "Lectures on Algebraic Topology," Springer-Verlag, Berlin, 1972.

4. S. MARDEŠIĆ AND I. SEgal, "Shape Theory," North Holland, Amsterdam, 1982.

5. J. Sanjurjo, Shape morphisms and small multivalued maps, Math. Japonica 35 (1990), 713-717.

6. J. Savjurjo, An intrinsic description of shape, Trans. Amer. Math. Soc. 329 (1992), 625-636.

Kopernikova 7

41020 Zagreb

CROATIA

Rebut el 12 de Gener de 1993 